\title{
A recursive condition for the symmetric nonnegative inverse eigenvalue problem
}

\author{
Elvis Ronald Valero ${ }^{a *}$, Exequiel Mallea-Zepeda ${ }^{a}$, \\ EBER LENES ${ }^{b}$ \\ ${ }^{a}$ Universidad de Tarapacá, Departamento de Matemática, Arica, Chile. \\ ${ }^{b}$ Universidad del Sinú. Elías Bechara Zainum, Departamento de Matemática, \\ Cartagena, Colombia.
}

\begin{abstract}
In this paper we present a sufficient condition and a necessary condition for Symmetric Nonnegative Inverse Eigenvalue Problem. This condition is independent of the existing realizability criteria. This criterion is recursive, that is, it determines whether a list $\Lambda=\left\{\lambda_{1}, \ldots, \lambda_{n}, \lambda_{n+1}\right\}$ is realizable by a nonnegative symmetric matrix, if the list $\mu=\left\{\mu_{1}, \ldots, \mu_{n}\right\}$ associated to $\Lambda$ is realizable. This result is easy to program and improves some existing criteria.

Keywords: Inverse problems, eigenvalues, orthogonal matrices, symmetric matrix.

MSC2010: 15A29, 15A18, 15B10, 15A57.

\section{Una condición recursiva para el problema inverso del autovalor para matrices simétricas no negativas}

\begin{abstract}
En este artículo presentamos una condición suficiente y una condición necesaria para el Problema Inverso de Autovalores para Matrices Simétricas no Negativas. Esta condición es independiente de los criterios de realizabilidad existentes. Este criterio es recursivo, es decir determina si una lista $\Lambda=\left\{\lambda_{1}, \ldots, \lambda_{n}, \lambda_{n+1}\right\}$ es realizable por una matriz simétrica no negativa, si la lista $\mu=\left\{\mu_{1}, \ldots, \mu_{n}\right\}$ asociada a $\Lambda$ es realizable. Este resultado es fácil de programar y mejora algunos criterios existentes.
\end{abstract}

Palabras clave: Problemas inversos, autovalores, matrices ortogonales, matrices simétricas.

\footnotetext{
${ }^{*}$ E-mail: evalero@uta.cl

Received: 9 March 2017, Accepted: 24 May 2017.

To cite this article: E.R. Valero, E. Mallea-Zepeda, E. Lenes, A recursive condition for the symmetric nonnegative inverse eigenvalue problem, Rev. Integr. Temas Mat. 35 (2017), No. 1, 37-50.
} 


\section{Introduction}

The nonnegative inverse eigenvalue problem (NIEP) is the problem of finding necessary and sufficient conditions for a list $\Lambda=\left\{\lambda_{1}, \lambda_{2}, \ldots, \lambda_{n}\right\}$ of complex numbers to be the spectrum of a $n \times n$ nonnegative matrix. The problem of finding necessary and sufficient conditions for a list of real numbers to be spectrum of a nonnegative matrix is called the real nonnegative inverse eigenvalue problem (RNIEP). Particularly, the problem of finding necessary and sufficient conditions for a list of real numbers $\Lambda$, to be the spectrum of a nonnegative symmetric matrix is called symmetric nonnegative inverse eigenvalue problem (SNIEP). These problems remain unsolved.

The NIEP, RNIEP and SNIEP are completely solved for $n \leq 4$. The NIEP has been solved for $n=3$ in 1978 by Loewy and London [7], for $n=4$ was solved by Meehan [9] in 1998, and subsequently independently in a different formulation by Torre-Mayo and others [17] in 2007.

The SNIEP has been solved when $n=3$ by Fiedler [2] in 1974 , and for $n=4$ has been solved by Guo [3] in 1996. The RNIEP and SNIEP are equivalent for $n \leq 4$ [3], but are different otherwise [5]. In 2011 Spector gives a complete solution to SNIEP when $n=5$ and the trace of nonnegative matrix is zero [16]. Partial results for the SNIEP have been obtained in [1], [2], [6], [8], [10], [11], [12], [13], [15].

This paper is organized as follows: In Section 2 we establish the notation and basic results in relation to SNIEP. In Section 3 we present the main results, a sufficient condition and a necessary condition. In Section 4, we show a programming algorithm in relation to the main results. The realizability criteria shown in Section 3 are independent of the criteria presented in [1], [13], and they improve those criteria.

\section{Notations and basic results}

Throughout this paper we use the following notation: Let $\mathbb{R}^{m \times n}$ the matrix set of order $m \times n$ with entries real numbers, in particular $\mathbb{R}^{n \times n}$ square matrices of order $n$. We denote $\rho(A)$ be the spectral radius of $A \in \mathbb{R}^{n \times n}$. We say that $A=\left[a_{i j}\right] \in \mathbb{R}^{n \times n}$ is nonnegative if $a_{i j} \geq 0$ for all $i, j \in\{1,2, \ldots, n\}$. We shall say that $\Lambda=\left\{\lambda_{1}, \lambda_{2}, \ldots, \lambda_{n}\right\}$ is realizable if there exists an nonnegative matrix $A \in \mathbb{R}^{n \times n}$ with spectrum $\Lambda$ and $\rho(A)=\lambda_{1}$. If $\Lambda$ is realizable for $A$, then it is said that $A$ realiza $\Lambda$.

If $\Lambda=\left\{\lambda_{1}, \lambda_{2}, \ldots, \lambda_{n+1}\right\}$ and $\mu=\left\{\mu_{1}, \mu_{2}, \ldots, \mu_{n}\right\}$ such that

$$
\lambda_{1} \geq \mu_{1} \geq \lambda_{2} \geq \ldots \geq \mu_{n-1} \geq \lambda_{n} \geq \mu_{n} \geq \lambda_{n+1},
$$

we define the functions $f(t)=\prod_{k=1}^{n+1}\left(t-\lambda_{k}\right), g(t)=\prod_{k=1}^{n}\left(t-\mu_{k}\right)$.

Show that the vector $\mathbf{y}=\left[\begin{array}{llll}y_{1} & y_{2} & \cdots & y_{n}\end{array}\right]^{T}$, with 


$$
y_{i}^{2}=-\frac{f\left(\mu_{i}\right)}{g^{\prime}\left(\mu_{i}\right)}=-\frac{\prod_{k=1}^{n+1}\left(\mu_{i}-\lambda_{k}\right)}{\prod_{k=1}^{i-1}\left(\mu_{i}-\mu_{k}\right) \cdot \prod_{k=i+1}^{n}\left(\mu_{i}-\mu_{k}\right)}
$$

is well defined (see [4]).

Finally, we present three results, the first two results are due to Horn and Johnson [4], and the third result was presented by Guo [3]. These will be later used for the development of new necessary and sufficient conditions for SNIEP.

Theorem 2.1. Let $\bar{A} \in \mathbb{R}^{n \times n}$ be a given symmetric matrix with eigenvalues $\mu_{1}, \ldots, \mu_{n}$, let $\mathbf{z} \in \mathbb{R}^{n \times 1}$ be a given vector (column matrix), and let $\alpha$ be a given real number. Let $A \in \mathbb{R}^{n+1 \times n+1}$ be the symmetric matrix obtained by bordering $\bar{A}$ with $\mathbf{z}$ and $\alpha$ as follows: $A=\left[\begin{array}{cc}\bar{A} & \mathbf{z} \\ \mathbf{z}^{T} & \alpha\end{array}\right]$, with eigenvalue $\lambda_{1}, \lambda_{2}, \ldots, \lambda_{n+1}$. If $\lambda_{1} \geq \lambda_{2} \geq \ldots \geq \lambda_{n+1}$ and $\mu_{1} \geq \mu_{2} \geq \ldots \geq \mu_{n}$. Then

$$
\lambda_{1} \geq \mu_{1} \geq \lambda_{2} \geq \ldots \geq \lambda_{n} \geq \mu_{n} \geq \lambda_{n+1} .
$$

Theorem 2.2. Let $n$ be a given positive integer, and let $\mu=\left\{\mu_{1}, \ldots, \mu_{n}\right\}$ and $\Lambda=$ $\left\{\lambda_{1}, \lambda_{2}, \ldots, \lambda_{n+1}\right\}$ be two lists of real numbers arranged in descending order such that

$$
\lambda_{1} \geq \mu_{1} \geq \lambda_{2} \geq \ldots \geq \lambda_{n} \geq \mu_{n} \geq \lambda_{n+1} .
$$

Let $D=\operatorname{diag}\left(\mu_{1}, \ldots, \mu_{n}\right)$. Then there exists a real number $\alpha$ and real vector $\mathbf{y} \in \mathbb{R}^{n \times 1}$ such that $\Lambda=\left\{\lambda_{1}, \lambda_{2}, \ldots, \lambda_{n+1}\right\}$ is the set of eigenvalues of the real symmetric matrix

$$
A=\left[\begin{array}{cc}
D & \mathbf{y} \\
\mathbf{y}^{T} & a
\end{array}\right]
$$

Theorem 2.3. The list $\lambda=\left\{\lambda_{1}, \lambda_{2}, \lambda_{3}, \lambda_{4}\right\}$ of real numbers is a realizable symmetric matrix if, and only if $\lambda_{1}+\lambda_{2}+\lambda_{3}+\lambda_{4} \geq 0$ and $\lambda_{1} \geq\left|\lambda_{i}\right|$ for $i=2,3,4$.

Observation: The matrix $A$ of Theorem 2.2 is similar to the matrix $\widetilde{A}=\left[\begin{array}{cc}a_{11} & z^{T} \\ z & D\end{array}\right]$, which has a spectrum $\Lambda$ and a main submatrix with spectrum $\mu$. In consequence, the results of the following section can be reformulated in such a way that the matrix that realize $\Lambda$ has the form of $\widetilde{A}$.

\section{Sufficient condition and necessary condition}

We consider a list $\Lambda=\left\{\lambda_{1}, \lambda_{2}, \ldots, \lambda_{n}\right\}$ of real numbers, such that $\lambda_{1} \geq \lambda_{2} \geq \ldots \geq \lambda_{n}$. The next result is a sufficient condition for the SNIEP [14].

Theorem 3.1. Let $\Lambda=\left\{\lambda_{1}, \lambda_{2}, \ldots, \lambda_{n+1}\right\}, \mu=\left\{\mu_{1}, \mu_{2}, \ldots, \mu_{n}\right\}$ be lists of real numbers, such that $\lambda_{1} \geq \mu_{1} \geq \lambda_{2} \geq \mu_{2} \geq \ldots \geq \mu_{n} \geq \lambda_{n+1}$, with $\sum_{k=1}^{n+1} \lambda_{k}-\sum_{k=1}^{n} \mu_{k} \geq 0$. Moreover let

Vol. 35, No. 1, 2017] 
$P$ be an orthogonal matrix, $D=\operatorname{diag}\left\{\mu_{1}, \ldots, \mu_{n}\right\}$ such that $P D P^{T} \geq 0$, and $\mathbf{y} \in \mathbb{R}^{n+1}$ given as (*) such that $P \mathbf{y} \geq 0$. Then there exists a nonnegative symmetric matrix $A$ with spectrum $\Lambda$.

Proof. We define $\mathbf{y}=\left[\begin{array}{lll}y_{1} & \ldots & y_{n}\end{array}\right]$ where each component $y_{i}$ is given as $(*)$, that is:

$$
y_{i}^{2}=-\frac{\prod_{k=1}^{n+1}\left(\mu_{i}-\lambda_{k}\right)}{\prod_{k=1}^{i-1}\left(\mu_{i}-\mu_{k}\right) \cdot \prod_{k=i+1}^{n}\left(\mu_{i}-\mu_{k}\right)} .
$$

By Theorem 2.1 the matrix $\bar{A}=\left[\begin{array}{cc}D & \mathbf{y} \\ \mathbf{y}^{T} & \sum_{k=1}^{n+1} \lambda_{k}-\sum_{k=1}^{n} \mu_{k}\end{array}\right]$ has spectrum $\Lambda$.

We define the orthogonal matrix $\left[\begin{array}{cc}P & \mathbf{0} \\ \mathbf{0}^{T} & 1\end{array}\right]$, with $\mathbf{0} \in \mathbb{R}^{n \times 1}$. Since $P D P^{T} \geq 0, P \mathbf{y} \geq 0$, then the symmetric matrix

$$
\begin{aligned}
A & =\left[\begin{array}{cc}
P & \mathbf{0} \\
\mathbf{0}^{T} & 1
\end{array}\right]\left[\begin{array}{cc}
D & \mathbf{y} \\
\mathbf{y}^{T} & \sum_{k=1}^{n+1} \lambda_{k}-\sum_{k=1}^{n} \mu_{k}
\end{array}\right]\left[\begin{array}{ll}
P^{T} & \mathbf{0} \\
\mathbf{0}^{T} & 1
\end{array}\right] \\
& =\left[\begin{array}{cc}
P D P^{T} & P \mathbf{y} \\
(P \mathbf{y})^{T} & \sum_{k=1}^{n+1} \lambda_{k}-\sum_{k=1}^{n} \mu_{k}
\end{array}\right]
\end{aligned}
$$

is nonnegative and with spectrum $\Lambda$.

The following example shows that Theorem 3.1 is independent of the realizability criteria established in [13, Lemma 4].

Example 3.2. Let $\Lambda=\{6,1,1,-4,-4\}$. For this list consider $\mu=\{4,1,-1,-4\}, a=2$. The matrix

$$
B=P \operatorname{diag}\{4,1,-1,4\} P^{T} \geq 0,
$$

where

$$
P=\left[\begin{array}{cccc}
\frac{2}{15} \sqrt{15} & -\frac{1}{30} \sqrt{210} & -\frac{1}{30} \sqrt{210} & \frac{2}{15} \sqrt{15} \\
\frac{8}{45} \sqrt{15} & -\frac{1}{90} \sqrt{210} & \frac{1}{90} \sqrt{210} & -\frac{8}{45} \sqrt{15} \\
\frac{1}{90} \sqrt{210} & \frac{8}{45} \sqrt{15} & -\frac{8}{45} \sqrt{15} & -\frac{1}{90} \sqrt{210} \\
\frac{1}{30} \sqrt{210} & \frac{2}{15} \sqrt{15} & \frac{2}{15} \sqrt{15} & \frac{1}{30} \sqrt{210}
\end{array}\right]
$$

have spectrum $\mu$.

[Revista Integración, temas de matemáticas 
We define $y=\left(\sqrt{\frac{48}{5}}, 0,-\sqrt{\frac{42}{5}}, 0\right)^{T}$; then, because of Theorem 3.1 the matrix

$$
\begin{aligned}
A & =\left[\begin{array}{ll}
P & 0 \\
0 & 1
\end{array}\right]\left[\begin{array}{ccc}
\operatorname{diag}(4,1,-1,4) & y \\
& y^{T} & 0
\end{array}\right]\left[\begin{array}{cc}
P^{T} & 0 \\
0 & 1
\end{array}\right] \\
& =\left[\begin{array}{ccccc}
0 & 3 & 0 & 0 & 3 \\
3 & 0 & 0 & \frac{2}{3} \sqrt{14} & \frac{5}{3} \\
0 & 0 & 0 & \frac{4}{3} & \frac{2}{3} \sqrt[3]{14} \\
0 & \frac{2}{3} \sqrt{14} & \frac{4}{3} & 0 & 0 \\
3 & \frac{5}{3} & \frac{2}{3} \sqrt[3]{14} & 0 & 0
\end{array}\right]
\end{aligned}
$$

is nonnegative with spectrum $\Lambda$.

The next example show that the Theorem 3.1 is independent of the realizability criteria shown in the [1, Theorem 3.4] and [13, Theorem 6].

Example 3.3. We consider the list $\Lambda=\{3+\sqrt{10}, 1,1,3-\sqrt{10},-4,-4\}$. For this list there exist a list $\mu=\{6,1,1,-4,-4\}$ and the orthogonal matrix

$$
P=\left[\begin{array}{ccccc}
\frac{\sqrt{7}}{5} & -\frac{\sqrt{10}}{10} & -\frac{\sqrt{14}}{10} & -\frac{\sqrt{10}}{5} & \frac{\sqrt{2}}{5} \\
\frac{\sqrt{2}}{5} & \frac{2 \sqrt{35}}{15} & \frac{2}{15} & -\frac{\sqrt{35}}{15} & -\frac{2 \sqrt{7}}{15} \\
\frac{\sqrt{7}}{5} & -\frac{2 \sqrt{10}}{15} & \frac{\sqrt{14}}{15} & \frac{\sqrt{10}}{15} & -\frac{7 \sqrt{2}}{15} \\
\frac{\sqrt{2}}{5} & 0 & \frac{4}{5} & 0 & \frac{\sqrt{7}}{5} \\
\frac{\sqrt{7}}{5} & \frac{\sqrt{10}}{5} & -\frac{\sqrt{14}}{10} & \frac{\sqrt{10}}{5} & \frac{\sqrt{2}}{5}
\end{array}\right],
$$

such that for $\boldsymbol{y}=\left[\begin{array}{lllll}1 & 0 & 0 & 0 & 0\end{array}\right]$, defined as (*) we have

$$
P \boldsymbol{y}=\left[\begin{array}{lllll}
\frac{1}{5} \sqrt{7} & \frac{1}{5} \sqrt{2} & \frac{1}{5} \sqrt{7} & \frac{1}{5} \sqrt{2} & \frac{1}{5} \sqrt{7}
\end{array}\right]^{T} \geq 0 .
$$

Thus, by Theorem 3.1, with $D=\operatorname{diag}\{6,1,1,-4,-4\}$, the matrix

Vol. 35, No. 1, 2017] 


$$
\begin{aligned}
A= & {\left[\begin{array}{ccccc}
P D P^{T} & \multicolumn{2}{c}{P \boldsymbol{y}} \\
(P \boldsymbol{y})^{T} & \sum_{i=1}^{6} \lambda_{i}-\sum_{i=1}^{5} \mu_{i}
\end{array}\right] } \\
= & {\left[\begin{array}{cccccc}
0 & 0 & 3 & 0 & 3 & \frac{1}{5} \sqrt{7} \\
0 & 0 & 0 & \frac{4}{3} & \frac{2}{3} \sqrt{14} & \frac{1}{5} \sqrt{2} \\
3 & 0 & 0 & \frac{2}{3} \sqrt{14} & \frac{5}{3} & \frac{1}{5} \sqrt{7} \\
0 & \frac{4}{3} & \frac{2}{3} \sqrt{14} & 0 & 0 & \frac{1}{5} \sqrt{2} \\
3 & \frac{2}{3} \sqrt{14} & \frac{5}{3} & 0 & 0 & \frac{1}{5} \sqrt{7} \\
\frac{1}{5} \sqrt{7} & \frac{1}{5} \sqrt{2} & \frac{1}{5} \sqrt{7} & \frac{1}{5} \sqrt{2} & \frac{1}{5} \sqrt{7} & 0
\end{array}\right] \geq 0, }
\end{aligned}
$$

has desired eigenvalues.

These examples show that Theorem 3.1 improves the realizability criteria presented in [1, Theorem 3.4] and [13, Lemma 4 and Theorem 6], in the sense that the lists $\{6,1,1,-4,-4\},\{3+\sqrt{10}, 1,1,3-\sqrt{10},-4,-4\}$ are not realizable by those criteria.

The next result shows a necessary condition for the SNIEP.

Theorem 3.4. Let $\Lambda=\left\{\lambda_{1}, \lambda_{2}, \ldots, \lambda_{n+1}\right\}$ realizable for a nonnegative symmetric matrix A. Then there exists:

1. A list of real number $\mu=\left\{\mu_{1}, \ldots, \mu_{n}\right\}$, such that $\lambda_{1} \geq \mu_{1} \geq \lambda_{2} \geq \mu_{2} \geq \ldots \geq \mu_{n} \geq$ $\lambda_{n+1}$.

2. A orthogonal matrix $Q$ and $\mathbf{b} \in \mathbb{R}^{n \times 1}$ such that $Q \mathbf{b} \geq 0$, and $Q D Q^{T} \geq 0$, where $D=\operatorname{diag}\left\{\mu_{1}, \ldots, \mu_{n}\right\}$.

Also it holds $\sum_{k=1}^{n+1} \lambda_{k}-\sum_{k=1}^{n} \mu_{k} \geq 0$.

Proof. Let $A$ be a nonnegative symmetric matrix with eigenvalues $\Lambda$. Without loss of generality we can assume that $A=\left[\begin{array}{cc}B & \mathbf{z} \\ \mathbf{z}^{T} & \alpha\end{array}\right]$, with $B \in \mathbb{R}^{n \times n}, \mathbf{z} \in \mathbb{R}^{n \times 1}$. Let $\mu=\sigma(B)=\left\{\mu_{1}, \ldots, \mu_{n}\right\}$. By Theorem 2.1 we get that

$$
\lambda_{1} \geq \mu_{1} \geq \lambda_{2} \geq \mu_{2} \geq \ldots \geq \mu_{n} \geq \lambda_{n+1} .
$$

Since $B$ is a nonnegative symmetric matrix, there exits a orthogonal matrix $Q$ such that $B=Q D Q^{T} \geq 0$. Let $\mathbf{b}=Q^{T} \mathbf{z}$. Since $\mathbf{z} \geq 0$ then we have that $Q \mathbf{b}=Q\left(Q^{T} \mathbf{z}\right)=\mathbf{z} \geq 0$. 
The matrix

$$
\hat{A}=\left[\begin{array}{cc}
D & Q^{T} \mathbf{z} \\
\left(Q^{T} \mathbf{z}\right)^{T} & \alpha
\end{array}\right]
$$

is similar to $A$, then $\sigma(\hat{A})=\left\{\lambda_{1}, \ldots, \lambda_{n+1}\right\}$. Since $\hat{A}$ is a symmetric matrix, then eigenvalues and the diagonal entries satisfy the following relation:

$$
\sum_{k=1}^{n+1} \lambda_{k}=\sum_{k=1}^{n} \mu_{k}+\alpha
$$

SO,

$$
\sum_{k=1}^{n+1} \lambda_{k}-\sum_{k=1}^{n} \mu_{k}=\alpha \geq 0
$$

therefore $\sum_{k=1}^{n+1} \lambda_{k}-\sum_{k=1}^{n} \mu_{k} \geq 0$.

Note that if $P \mathbf{y}=\mathbf{z}$, with $P$ given in Theorem 3.1, $\mathbf{y}$ defined as $(*)$ and $\mathbf{z}$ given in Theorem 3.4, then Theorem 3.1 and Theorem 3.4 establish necessary and sufficient conditions for SNIEP.

Let $\Lambda=\left\{\lambda_{1}, \ldots, \lambda_{n}\right\}$ a list of real numbers. If $n=2,3$, it is easy to see that $P \mathbf{y}=\mathbf{z}$. For $n=4$, with some ideas presented in [3], we consider the following cases:

1. If $\lambda_{1} \geq \lambda_{2} \geq \lambda_{3} \geq \lambda_{4}>0$, then the symmetric matrix

$$
A_{1}=\operatorname{diag}\left\{\lambda_{1}, \lambda_{2}, \lambda_{3}, \lambda_{4}\right\}
$$

has eigenvalues $\Lambda$. In this case we have $\mu=\left\{\lambda_{1}, \lambda_{2}, \lambda_{3}\right\}, P \mathbf{y}=\mathbf{z}$ with $P=I \in$ $\mathbb{R}^{3 \times 3}$, and $\mathbf{y}=\mathbf{z}=\left[\begin{array}{lll}0 & 0 & 0\end{array}\right]^{T}$.

2. If $\lambda_{1} \geq \lambda_{2} \geq \lambda_{3} \geq 0>\lambda_{4}$, then the symmetric matrix

$$
A_{2}=\left[\begin{array}{cc}
B_{1} & O \\
O & B_{2}
\end{array}\right]
$$

with

$$
B_{1}=\left[\begin{array}{ll}
\frac{\lambda_{1}+\lambda_{4}}{2} & \frac{\lambda_{1}-\lambda_{4}}{2} \\
\frac{\lambda_{1}-\lambda_{4}}{2} & \frac{\lambda_{1}+\lambda_{4}}{2}
\end{array}\right], B_{2}=\left[\begin{array}{cc}
\lambda_{2} & 0 \\
0 & \lambda_{3}
\end{array}\right] \text { and } O=\left[\begin{array}{cc}
0 & 0 \\
0 & 0
\end{array}\right] \text {, }
$$

has spectrum $\Lambda$. In this case we have $\mu=\left\{\lambda_{1}, \lambda_{2}, \lambda_{4}\right\}, P \mathbf{y}=\mathbf{z}$, with

$$
P=\left[\begin{array}{ccc}
\frac{1}{\sqrt{2}} & 0 & -\frac{1}{\sqrt{2}} \\
\frac{1}{\sqrt{2}} & 0 & \frac{1}{\sqrt{2}} \\
0 & 1 & 0
\end{array}\right] \text { and } \mathbf{y}=\mathbf{z}=\left[\begin{array}{l}
0 \\
0 \\
0
\end{array}\right]
$$

Vol. 35, No. 1, 2017] 
3. If $\lambda_{1} \geq \lambda_{2} \geq 0>\lambda_{3} \geq \lambda_{4}$ and $\lambda_{2} \geq\left|\lambda_{3}\right|$, the matrix

$$
A=\left[\begin{array}{cccc}
\frac{\lambda_{2}+\lambda_{3}}{2} & \frac{\lambda_{2}-\lambda_{3}}{2} & 0 & 0 \\
\frac{\lambda_{2}-\lambda_{3}}{2} & \frac{\lambda_{2}+\lambda_{3}}{2} & 0 & 0 \\
0 & 0 & \frac{\lambda_{1}+\lambda_{4}}{2} & \frac{\lambda_{1}-\lambda_{4}}{2} \\
0 & 0 & \frac{\lambda_{1}-\lambda_{4}}{2} & \frac{\lambda_{1}+\lambda_{4}}{2}
\end{array}\right]
$$

has spectrum $\Lambda$. In this case we have

$$
\begin{gathered}
\mu=\left\{\lambda_{2}, \lambda_{3}, \frac{\lambda_{1}+\lambda_{4}}{2}\right\}, P=\left[\begin{array}{ccc}
\frac{1}{\sqrt{2}} & 0 & -\frac{1}{\sqrt{2}} \\
\frac{1}{\sqrt{2}} & 0 & \frac{1}{\sqrt{2}} \\
0 & 1 & 0
\end{array}\right], \\
\mathbf{y}=\left[\begin{array}{c}
0 \\
\frac{\lambda_{1}-\lambda_{4}}{2} \\
0
\end{array}\right] \text { and } \mathbf{z}=\left[\begin{array}{c}
0 \\
0 \\
\frac{\lambda_{1}-\lambda_{4}}{2}
\end{array}\right],
\end{gathered}
$$

and so, $P \mathbf{y}=\mathbf{z}$.

The case $\lambda_{1} \geq \lambda_{2} \geq 0>\lambda_{3} \geq \lambda_{4}$ and $\lambda_{2}<\left|\lambda_{3}\right|$ is analogous to the previous case.

4. If $\lambda_{1} \geq 0>\lambda_{2} \geq \lambda_{3} \geq \lambda_{4}$, the matrix $\left[\begin{array}{ll}A_{1} & \mathbf{z} \\ \mathbf{z}^{T} & 0\end{array}\right]$ has eigenvalues $\Lambda$, with

$$
\begin{aligned}
& \mathbf{z}=\left[\begin{array}{lll}
\sqrt{\frac{-\lambda_{1} \lambda_{2}\left(\lambda_{1}+\lambda_{2}\right)}{2\left(\lambda_{1}+\lambda_{2}-\lambda_{3}\right)}} & \sqrt{\frac{-\lambda_{1} \lambda_{2}\left(\lambda_{1}+\lambda_{2}\right)}{2\left(\lambda_{1}+\lambda_{2}-\lambda_{3}\right)}} & \sqrt{\frac{\lambda_{1} \lambda_{2} \lambda_{3}}{\lambda_{1}+\lambda_{2}-\lambda_{3}}}
\end{array}\right]^{T}, \\
& A_{1}=\left[\begin{array}{lll}
\frac{\lambda_{1}+\lambda_{2}+\lambda_{3}+\lambda_{4}}{2} & \frac{\lambda_{1}+\lambda_{2}+\lambda_{3}-\lambda_{4}}{2} & \sqrt{\frac{-\lambda_{3}\left(\lambda_{1}+\lambda_{2}\right)}{2}} \\
\frac{\lambda_{1}+\lambda_{2}+\lambda_{3}-\lambda_{4}}{2} & \frac{\lambda_{1}+\lambda_{2}+\lambda_{3}+\lambda_{4}}{2} & \sqrt{\frac{-\lambda_{3}\left(\lambda_{1}+\lambda_{2}\right)}{2}} \\
\sqrt{\frac{-\lambda_{3}\left(\lambda_{1}+\lambda_{2}\right)}{2}} & \sqrt{\frac{-\lambda_{3}\left(\lambda_{1}+\lambda_{2}\right)}{2}} & 0
\end{array}\right] .
\end{aligned}
$$

In this case we have $\mu=\left\{\lambda_{1}+\lambda_{2}, \lambda_{3}, \lambda_{4}\right\}$,

$$
P=\left[\begin{array}{ccc}
\sqrt{\frac{\lambda_{1}+\lambda_{2}}{2\left(\lambda_{1}+\lambda_{2}-\lambda_{3}\right)}} & -\sqrt{\frac{-\lambda_{3}}{2\left(\lambda_{1}+\lambda_{2}-\lambda_{3}\right)}} & -\frac{1}{\sqrt{2}} \\
\sqrt{\frac{\lambda_{1}+\lambda_{2}}{2\left(\lambda_{1}+\lambda_{2}-\lambda_{3}\right)}} & -\sqrt{\frac{-\lambda_{3}}{2\left(\lambda_{1}+\lambda_{2}-\lambda_{3}\right)}} & \frac{1}{\sqrt{2}} \\
\sqrt{\frac{-\lambda_{3}}{\lambda_{1}+\lambda_{2}-\lambda_{3}}} & \sqrt{\frac{\lambda_{1}+\lambda_{2}}{\lambda_{1}+\lambda_{2}-\lambda_{3}}} & 0
\end{array}\right], \mathbf{y}=\left[\begin{array}{c}
\sqrt{-\lambda_{1} \lambda_{2}} \\
0 \\
0
\end{array}\right],
$$

and it holds that $P \mathbf{y}=\mathbf{z}$.

We conjecture that the Theorem 3.4 and Theorem 3.1 establish a necessary and sufficient condition for $S N I E P$ when $n \geq 5$, that is, it holds $P \mathbf{y}=\mathbf{z}$, with $P$ given as in Theorem $3.1, \mathbf{y}$ given as $(*)$ and $\mathbf{z}$ given as in the Theorem 3.4.

The SNIEP for a list $\Lambda=\left\{\lambda_{1}, \lambda_{2}, \ldots, \lambda_{n}\right\}$ is equivalent to find an orthogonal $n$-dimensional matrix $P$, such that $P D_{\Lambda} P^{T} \geq 0$, where $D_{\Lambda}=\operatorname{diag}\left\{\lambda_{1}, \lambda_{2}, \ldots, \lambda_{n}\right\}$.

We consider the following orthogonal matrices:

[Revista Integración, temas de matemáticas 
1.

$$
P_{1}=\left[\begin{array}{cccc}
\frac{1}{\sqrt{2}} & 0 & 0 & -\frac{1}{\sqrt{2}} \\
\frac{1}{\sqrt{2}} & 0 & 0 & \frac{1}{\sqrt{2}} \\
0 & 1 & 0 & 0 \\
0 & 0 & 1 & 0
\end{array}\right], P_{2}=\left[\begin{array}{cccc}
0 & \frac{1}{\sqrt{2}} & -\frac{1}{\sqrt{2}} & 0 \\
0 & \frac{1}{\sqrt{2}} & \frac{1}{\sqrt{2}} & 0 \\
\frac{1}{\sqrt{2}} & 0 & 0 & -\frac{1}{\sqrt{2}} \\
\frac{1}{\sqrt{2}} & 0 & 0 & \frac{1}{\sqrt{2}}
\end{array}\right]
$$

2. For the list of real numbers $\mu=\left\{\mu_{1}, \mu_{2}, \mu_{3}, \mu_{4}\right\}$ such that $\mu_{1} \geq\left|\lambda_{i}\right|$, for $i=2,3,4$, $\mu_{3}<0$ and $\mu_{2}<\left|\mu_{3}\right|$, we define

$$
P_{3}=\left[\begin{array}{cccc}
\frac{\sqrt{\mu_{1}+\mu_{3}}}{\sqrt{2} \sqrt{\mu_{1}-\mu_{2}}} & -\frac{\sqrt{-\left(\mu_{2}+\mu_{3}\right)}}{\sqrt{2} \sqrt{\mu_{1}-\mu_{2}}} & 0 & -\frac{1}{\sqrt{2}} \\
\frac{\sqrt{\mu_{1}+\mu_{3}}}{\sqrt{2} \sqrt{\mu_{1}-\mu_{2}}} & -\frac{\sqrt{-\left(\mu_{2}+\mu_{3}\right)}}{\sqrt{2} \sqrt{\mu_{1}-\mu_{2}}} & 0 & \frac{1}{\sqrt{2}} \\
\frac{\sqrt{-\left(\mu_{2}+\mu_{3}\right)}}{\sqrt{2} \sqrt{\mu_{1}-\mu_{2}}} & \frac{\sqrt{\mu_{1}+\mu_{3}}}{\sqrt{2} \sqrt{\mu_{1}-\mu_{2}}} & -\frac{1}{\sqrt{2}} & 0 \\
\frac{\sqrt{-\left(\mu_{2}+\mu_{3}\right)}}{\sqrt{2} \sqrt{\mu_{1}-\mu_{2}}} & \frac{\sqrt{\mu_{1}+\mu_{3}}}{\sqrt{2} \sqrt{\mu_{1}-\mu_{2}}} & \frac{1}{\sqrt{2}} & 0
\end{array}\right]
$$

3. For the list of real numbers $\mu=\left\{\mu_{1}, \mu_{2}, \mu_{3}, \mu_{4}\right\}$ such that $\mu_{1} \geq\left|\lambda_{i}\right|$, for $i=2,3,4$ and $\mu_{2}<0$, we define

$$
P_{4}=\left[\begin{array}{cccc}
\frac{\sqrt{\mu_{1}} \sqrt{\mu_{1}+\mu_{2}}}{\sqrt{2} \sqrt{\mu_{1}+\mu_{2}-\mu_{3}} \sqrt{\mu_{1}-\mu_{2}}} & \frac{-\sqrt{-\mu_{2}} \sqrt{\mu_{1}+\mu_{2}}}{\sqrt{2} \sqrt{\mu_{1}+\mu_{2}-\mu_{3}} \sqrt{\mu_{1}-\mu_{2}}} & \frac{-\sqrt{-\mu_{3}}}{\sqrt{2} \sqrt{\mu_{1}+\mu_{2}-\mu_{3}}} & -\frac{1}{\sqrt{2}} \\
\frac{\sqrt{\mu_{1}} \sqrt{\mu_{1}+\mu_{2}}}{\sqrt{2} \sqrt{\mu_{1}+\mu_{2}-\mu_{3}} \sqrt{\mu_{1}-\mu_{2}}} & \frac{-\sqrt{-\mu_{2}} \sqrt{\mu_{1}+\mu_{2}}}{\sqrt{2} \sqrt{\mu_{1}+\mu_{2}-\mu_{3}} \sqrt{\mu_{1}-\mu_{2}}} & \frac{-\sqrt{-\mu_{3}}}{\sqrt{2} \sqrt{\mu_{1}+\mu_{2}-\mu_{3}}} & \frac{1}{\sqrt{2}} \\
\frac{\sqrt{\mu_{1}} \sqrt{-\mu_{3}}}{\sqrt{\mu_{1}+\mu_{2}-\mu_{3}} \sqrt{\mu_{1}-\mu_{2}}} & \frac{-\sqrt{-\mu_{2}} \sqrt{-\mu_{3}}}{\sqrt{\mu_{1}+\mu_{2}-\mu_{3}} \sqrt{\mu_{1}-\mu_{2}}} & \frac{\sqrt{\mu_{1}+\mu_{2}}}{\sqrt{\mu_{1}+\mu_{2}-\mu_{3}}} & 0 \\
\frac{\sqrt{-\mu_{2}}}{\sqrt{\mu_{1}-\mu_{2}}} & \frac{\sqrt{\mu_{1}}}{\sqrt{\mu_{1}-\mu_{2}}} & 0 & 0
\end{array}\right] .
$$

The following result gives a sufficient condition for realizability of list $\Lambda$ when $n=5$, by means of orthogonal matrices.

Corollary 3.5. Let $\Lambda=\left\{\lambda_{1}, \lambda_{2}, \lambda_{3}, \lambda_{4}, \lambda_{5}\right\}, \mu=\left\{\mu_{1}, \mu_{2}, \mu_{3}, \mu_{4}\right\}$ lists of real numbers such that $\lambda_{1} \geq \mu_{1} \geq \lambda_{2} \geq \mu_{2} \geq \lambda_{3} \geq \mu_{3} \geq \lambda_{4} \geq \mu_{4} \geq \lambda_{5} ; \sum_{i=1}^{5} \lambda_{i}-\sum_{i=1}^{4} \mu_{i} \geq 0 ; \mu_{1} \geq\left|\lambda_{i}\right|$ for $i=2,3,4$ and $\mathbf{y} \in \mathbb{R}^{4}$ defined as (*). If one of the following conditions is true:
1. $P_{1} \mathbf{y} \geq 0 \wedge \mu_{3} \geq 0>\mu_{4}$,
2. $P_{2} \mathbf{y} \geq 0 \wedge \mu_{2} \geq 0>\mu_{3} \wedge \mu_{2} \geq\left|\mu_{3}\right|$,
3. $P_{3} \mathbf{y} \geq 0 \wedge \mu_{2} \geq 0>\mu_{3} \wedge \mu_{2}<\left|\mu_{3}\right|$,

Vol. 35, No. 1, 2017] 
4. $P_{4} \mathbf{y} \geq 0 \wedge \mu_{1} \geq 0>\mu_{2}$,

then $\Lambda$ is the spectrum of a nonnegative symmetric matrix.

Proof. We recall that $\mathbf{y} \in \mathbb{R}^{4 \times 1}$ is defined by

$$
y_{i}=-\frac{\prod_{k=1}^{5}\left(\mu_{i}-\lambda_{k}\right)}{\prod_{k=1}^{i-1}\left(\mu_{i}-\mu_{k}\right) \cdot \prod_{k=i+1}^{4}\left(\mu_{i}-\mu_{k}\right)}
$$

We study the case (1): If $P_{1} \mathbf{y} \geq 0, \mu_{3} \geq 0>\mu_{4}$.

We define the symmetric matrix

$$
A=\left[\begin{array}{ccccc}
\frac{1}{\sqrt{2}} & 0 & 0 & -\frac{1}{\sqrt{2}} & 0 \\
\frac{1}{\sqrt{2}} & 0 & 0 & \frac{1}{\sqrt{2}} & 0 \\
0 & 1 & 0 & 0 & 0 \\
0 & 0 & 1 & 0 & 0 \\
0 & 0 & 0 & 0 & 1
\end{array}\right]\left[\begin{array}{ccccc}
\mu_{1} & 0 & 0 & 0 & y_{1} \\
0 & \mu_{2} & 0 & 0 & y_{2} \\
0 & 0 & \mu_{3} & 0 & y_{3} \\
0 & 0 & 0 & \mu_{4} & y_{4} \\
y_{1} & y_{2} & y_{3} & y_{4} & a
\end{array}\right]\left[\begin{array}{ccccc}
\frac{1}{\sqrt{2}} & \frac{1}{\sqrt{2}} & 0 & 0 & 0 \\
0 & 0 & 1 & 0 & 0 \\
0 & 0 & 0 & 1 & 0 \\
-\frac{1}{\sqrt{2}} & \frac{1}{\sqrt{2}} & 0 & 0 & 0 \\
0 & 0 & 0 & 0 & 1
\end{array}\right],
$$

that is

$$
A=\left[\begin{array}{ccccc}
\frac{1}{2} \mu_{1}+\frac{1}{2} \mu_{4} & \frac{1}{2} \mu_{1}-\frac{1}{2} \mu_{4} & 0 & 0 & \frac{1}{2} \sqrt{2} y_{1}-\frac{1}{2} \sqrt{2} y_{4} \\
\frac{1}{2} \mu_{1}-\frac{1}{2} \mu_{4} & \frac{1}{2} \mu_{1}+\frac{1}{2} \mu_{4} & 0 & 0 & \frac{1}{2} \sqrt{2} y_{1}+\frac{1}{2} \sqrt{2} y_{4} \\
0 & 0 & \mu_{2} & 0 & y_{2} \\
0 & 0 & 0 & \mu_{3} & y_{3} \\
\frac{1}{2} \sqrt{2} y_{1}-\frac{1}{2} \sqrt{2} y_{4} & \frac{1}{2} \sqrt{2} y_{1}+\frac{1}{2} \sqrt{2} y_{4} & y_{2} & y_{3} & a
\end{array}\right],
$$

whit $a=\sum_{i=1}^{5} \lambda_{i}-\sum_{i=1}^{4} \mu_{i} \geq 0$.

Since, by hypothesis $\mu_{1} \geq\left|\mu_{i}\right|, i=2,3,4$, we have $\frac{1}{2} \mu_{1} \pm \frac{1}{2} \mu_{4} \geq 0$. Moreover, since $\mu_{3} \geq 0$, then $\mu_{2} \geq 0$. Therefore the matrix $A$ is nonnegative.

Also

$$
P_{1} \mathbf{y}=\left[\begin{array}{llll}
\frac{1}{2} \sqrt{2} y_{1}-\frac{1}{2} \sqrt{2} y_{4} & \frac{1}{2} \sqrt{2} y_{1}+\frac{1}{2} \sqrt{2} y_{4} \quad y_{3} \quad y_{4}
\end{array}\right]^{T}
$$

by Theorem 3.1 the nonnegative symmetric matrix $A$ has spectrum $\Lambda$.

Otherwise it is similarly derived. 
Example 3.6. Consider the list $\Lambda=\{6,3,3,-5,-5\}$. If the list $\Lambda$ is realizable, then there exists a realizable list $\mu=\left\{\mu_{1}, \mu_{2}, \mu_{3}, \mu_{4}\right\}$. By theorems 2.1, 2.2, 2.3, we have $6 \geq \mu_{1} \geq 5, \mu_{2}=3,-1 \geq \mu_{3} \geq-4, \mu_{4}=-5$ and $4 \geq \mu_{1}+\mu_{3} \geq 2$.

On the other hand, for $\Lambda$ and $\mu$ define $\boldsymbol{y}=\left[\begin{array}{llll}y_{1} & y_{2} & y_{3} & y_{4}\end{array}\right]^{T}$, with $y_{i}, i=1,2,3,4$, as (*). There is not $P_{i}$ of the Corollary 3.5 such that $P_{i} \boldsymbol{y} \geq 0$, thus by Theorems 3.1, 3.4, the list $\Lambda=\{6,3,3,-5,-5\}$ is not realizable.

Observation: In [6] it is shown that this list can not be performed by any criterion.

\section{Algorithm}

In this section, we will implement an algorithm to decide whether a list $\Lambda=$ $\left\{\lambda_{1}, \lambda_{2}, \ldots, \lambda_{n}\right\}$ is the spectrum of a nonnegative symmetric matrix, by using the results presented in the previous section. We introduce the following notation:

$$
\begin{aligned}
\mathbb{O}_{n} & =\left\{P \in \mathbb{R}^{n \times n}: P P^{T}=P^{T} P=I\right\}, \\
\mathbb{S}_{n} & =\left\{\Lambda=\left\{\lambda_{1}, \ldots, \lambda_{n}\right\}: \exists A=A^{T}, \sigma(A)=\Lambda\right\}, \\
D_{\Lambda} & =\operatorname{diag}\left\{\lambda_{1}, \ldots, \lambda_{n}\right\}, \\
\mathbb{S}_{n}(\Lambda) & =\left\{A \geq 0: A=A^{T} \geq 0, \sigma(A)=\Lambda\right\}, \\
\mathbb{O}_{n}(\Lambda) & =\left\{P \in \mathbb{O}_{n}: P D_{\Lambda} P^{T} \geq 0\right\} .
\end{aligned}
$$

Note that $\mathbb{S}_{n}(\Lambda) \neq \emptyset$ if only if $\mathbb{O}_{n}(\Lambda) \neq \emptyset$, and if $\lambda_{n} \geq 0$ then $\mathbb{O}_{n}(\Lambda) \neq \emptyset$. Also $\Lambda \in \mathbb{S}_{n}$ if and only if $\mathbb{S}_{n}(\Lambda) \neq \emptyset$ or $\mathbb{O}_{n}(\Lambda) \neq \emptyset$.

\section{Algorithm}

1. Let $\Lambda=\left\{\lambda_{1}, \lambda_{2}, \ldots, \lambda_{n+1}\right\}$

2. Let $\mu=\left\{\mu_{1}, \ldots, \mu_{n}\right\}$ be such that $\lambda_{1} \geq \mu_{1} \geq \lambda_{2} \geq \ldots \geq \lambda_{n} \geq \mu_{n} \geq \lambda_{n+1}$, $\sum_{i=1}^{n+1} \lambda_{i}-\sum_{i=1}^{n} \mu_{i} \geq 0$ and $\sum_{i=1}^{n} \mu_{i} \geq 0$.

3. If $\mu \in \mathbb{S}_{n}$, define $\mathbf{y}=\left[\begin{array}{lll}y_{1} & \ldots & y_{n}\end{array}\right]^{T}$ as $(*)$. If else return step 2 .

4. Let $P \in \mathbb{O}_{n}(\mu)$.

5. If $P \mathbf{y} \geq 0$, define

$$
A=\left[\begin{array}{cc}
P D_{\mu} P^{T} & P \mathbf{y} \\
(P \mathbf{y})^{T} & \sum_{i=1}^{n+1} \lambda_{i}-\sum_{i=1}^{n} \mu_{i}
\end{array}\right] \in \mathbb{S}_{n}(\Lambda)
$$

if else, return to step 4.

Vol. 35, No. 1, 2017] 
Naturally there exists several ways to select the $\mu_{i}$, as well as several ways to determine if $\mu \in \mathbb{S}_{n}$. For the case $n=5$, the selection of $\mu$ is limited, since for $n=4$ there are necessary and sufficient conditions to determine if $\mu \in \mathbb{S}_{n}$.

The following example shows that the algorithm presented is recursive.

Example 4.1. We consider the list $\Lambda=\{9,1,-1,-2,-6\}$.

We select $\mu=\{6,-1,-2,-3\}$. To show that $\mu$ is realizable, select the list $\nu=\{3,-1,-2\}$.

For the list $\nu$, we define $\mathbf{x}^{T}=\left[\begin{array}{ccc}3 \sqrt{2} & 0 & 0\end{array}\right]$, and the orthogonal matrix

$$
P_{1}=\left[\begin{array}{ccc}
\frac{1}{10} \sqrt{30} & -\frac{1}{\sqrt{2}} & -\frac{\sqrt{5}}{5} \\
\frac{1}{10} \sqrt{30} & \frac{1}{\sqrt{2}} & -\frac{\sqrt{5}}{5} \\
\frac{1}{5} \sqrt{10} & 0 & \overline{5} \sqrt{15}
\end{array}\right] .
$$

Thus the matrix

$$
\begin{aligned}
& A_{1}=\left[\begin{array}{cc}
P_{1} & 0 \\
0 & 1
\end{array}\right]\left[\begin{array}{cc}
\operatorname{diag}\{3,-1,-2\} & \mathbf{x} \\
\mathbf{x}^{T} & 0
\end{array}\right]\left[\begin{array}{cc}
P_{1}^{T} & 0 \\
0 & 1
\end{array}\right] \\
& =\left[\begin{array}{cccc}
0 & 1 & \frac{1}{2} \sqrt{2} \sqrt{6} & \frac{3}{10} \sqrt{2} \sqrt{5} \sqrt{6} \\
1 & 0 & \frac{1}{2} \sqrt{2} \sqrt{6} & \frac{3}{10} \sqrt{2} \sqrt{5} \sqrt{6} \\
\frac{1}{2} \sqrt{2} \sqrt{6} & \frac{1}{2} \sqrt{2} \sqrt{6} & 0 & \frac{6}{5} \sqrt{5} \\
\frac{3}{10} \sqrt{2} \sqrt{5} \sqrt{6} & \frac{3}{10} \sqrt{2} \sqrt{5} \sqrt{6} & \frac{6}{5} \sqrt{5} & 0
\end{array}\right] \\
& =\left[\begin{array}{cccc}
0 & 1.0 & 1.7321 & 2.3238 \\
1.0 & 0 & 1.7321 & 2.3238 \\
1.7321 & 1.7321 & 0 & 2.6833 \\
2.3238 & 2.3238 & 2.6833 & 0
\end{array}\right]
\end{aligned}
$$

it is nonnegative symmetrical with spectrum $\mu$.

For the list $\mu$, we define $\mathbf{y}=\left[\begin{array}{llll}2 \sqrt{5} & 0 & 0 & 4\end{array}\right]^{T}$ and the orthogonal matrix

$$
P=\left[\begin{array}{cccc}
\frac{1}{5} \sqrt{5} & -\frac{1}{\sqrt{2}} & -\frac{1}{5} \sqrt{5} & -\frac{1}{30} \sqrt{90} \\
\frac{1}{5} \sqrt{5} & \frac{1}{\sqrt{2}} & -\frac{1}{5} \sqrt{5} & -\frac{1}{30} \sqrt{90} \\
\frac{2}{15} \sqrt{15} & 0 & \frac{1}{5} \sqrt{15} & -\frac{1}{15} \sqrt{30} \\
\frac{1}{3} \sqrt{3} & 0 & 0 & \frac{1}{3} \sqrt{6}
\end{array}\right] .
$$

Thus the matrix 


$$
\begin{aligned}
& A=\left[\begin{array}{ll}
P & 0 \\
0 & 1
\end{array}\right]\left[\begin{array}{cc}
\operatorname{diag}\{6,-1,-2,-3\} & \mathbf{y} \\
\mathbf{y}^{T} & 0
\end{array}\right]\left[\begin{array}{cc}
P^{T} & 0 \\
0 & 1
\end{array}\right] \\
& =\left[\begin{array}{ccccc}
0 & 1 & \frac{1}{2} \sqrt{12} & \frac{3}{10} \sqrt{60} & 2-\frac{2 \sqrt{90}}{15} \\
1 & 0 & \frac{1}{2} \sqrt{12} & \frac{3}{10} \sqrt{60} & 2-\frac{2 \sqrt{90}}{15} \\
\frac{1}{2} \sqrt{12} & \frac{1}{2} \sqrt{12} & 0 & \frac{6}{5} \sqrt{5} & \frac{4}{3} \sqrt{3}-\frac{4 \sqrt{30}}{15} \\
\frac{3}{10} \sqrt{60} & \frac{3}{10} \sqrt{60} & \frac{6}{5} \sqrt{5} & 0 & \frac{4}{3} \sqrt{6}+\frac{2 \sqrt{15}}{3} \\
2-\frac{2}{15} \sqrt{90} & 2-\frac{2}{15} \sqrt{90} & \frac{4}{3} \sqrt{3}-\frac{4}{15} \sqrt{30} & \frac{4}{3} \sqrt{6}+\frac{2 \sqrt{15}}{3} & 1
\end{array}\right] \\
& =\left[\begin{array}{ccccc}
0 & 1.0 & 1.7321 & 2.3238 & 0.73509 \\
1.0 & 0 & 1.7321 & 2.3238 & 0.73509 \\
1.7321 & 1.7321 & 0 & 2.6833 & 0.84881 \\
2.3238 & 2.3238 & 2.6833 & 0 & 5.8480 \\
0.73509 & 0.73509 & 0.84881 & 5.8480 & 1.0
\end{array}\right]
\end{aligned}
$$

it is nonnegative symmetrical with spectrum $\Lambda=\{9,1,-1,-2,-6\}$.

\section{Acknowledgement}

This work was supported by Proyecto UTA-Mayor, 4735-16 of the Universidad de Tarapacá, Arica-Chile.

\section{References}

[1] Ellard R. and Smigoc H., "Connecting sufficient conditions for the Symmetric Nonnegative Inverse Eigenvalues Problem", Linear Algebra Appl. 498 (2016), 521-552.

[2] Fiedler M., "Eigenvalues of Nonnegative Symmetric Matrices", Linear Algebra Appl. 9 (1974), 119-142.

[3] Guo W., "An Inverse Eigenvalues Problem for Nonnegative Matrices", Linear Algebra Appl. 249 (1996), No. 1-3, 67-78.

[4] Horn A. and Johnson C.R., Matrix analysis, Cambridge University Press, Cambridge, 1990. Corrected reprint of the 1985 original.

[5] Johnson C.R., Laffey T.J. and Loewy R., "The real and the symmetric nonnegative inverse eigenvalue problems are different", Proc. Amer. Math. Soc. 124 (1996), No. 12, 3647-3651.

[6] Johnson C.R., Marijuán C. and Pisonero M., "Ruling out certain 5-spectra for the symmetric nonnegative inverse eigenvalue problem", Linear Algebra Appl. 512 (2017), 129-135.

[7] Loewy R. and London D., "A note on an inverse problem for nonnegative matrices", Linear Multilinear Algebra 6 (1978), No. 1, 83-90.

Vol. 35, No. 1, 2017] 
[8] Loewy R. and McDonald J.J., "The symmetric nonnegative inverse eigenvalue problem for $5 \times 5$ matrices", Linear Algebra Appl. 393 (2004), 275-298.

[9] Meehan M.E., "Some results on the matrix spectra", Thesis (Ph.D.), National University of Ireland, Dublin, 1998.

[10] McDonald J.J. and Neumann M., "The Soules approach to the inverse eigenvalues problem for nonnegative symmetric matrices of order $n \leq 5$ ", in Contemp. Math. 259, Amer. Math. Soc.(2000), 387-407.

[11] Radwan N., "An Inverse eigenvalue problem for symmetric and normal matrices", Linear Algebra Appl. 248 (1996), 101-109.

[12] Soto R.L., "A family of realizability criteria for the real and symmetric nonnegative inverse eigenvalue problem", Numer. Linear Algebra Appl. 20 (2013), No. 2, 336-348.

[13] Soto R.L., "Realizability criterion for the symmetric nonnegative inverse eigenvalue problem", Linear Algebra Appl. 416 (2006), No. 2-3, 783-794.

[14] Soto R.L. and Valero E., "On Symmetric Nonnegative matrices with Prescribed Spectrum", International Mathematical Forum 9 (2014), No. 24, 1161-1176.

[15] Soules G.,"Constructing symmetric nonnegative matrices", Linear Multilinear Algebra 13 (1983), No. 3, 241-251.

[16] Spector O., "A characterization of trace zero symmetric nonnegative $5 \times 5$ matrices", Linear Algebra Appl. 434 (2011), No. 4, 1000-1017.

[17] Torre-Mayo J., Abril-Raymundo M.R., Alarcia-Estévez E., Marijuán C., and Pisonero M., "The nonnegative inverse eigenvalue problem from the coefficients of the characteristic polynomial. ELB digraphs", Linear Algebra Appl. 426 (2007), No. 2-3, 729-773. 